\title{
Dimensionamento de sistema de recirculação aquícola marinho piloto para engorda de peixes
}

\author{
Luiz Guilherme Buglione Peruch', Fabiano Muller Silva² e Katt Regina Lapa ${ }^{3}$
}

\begin{abstract}
Resumo - Os sistemas de recirculação aquícola (RAS) para o cultivo e manutenção de peixes marinhos são alternativos ao cultivo em tanques-rede. Neste sentido, esforços vêm sendo realizados para o desenvolvimento destes sistemas em escala comercial no Brasil. O objetivo do trabalho foi dimensionar e instalar em escala piloto laboratorial um RAS para produção de sardinha verdadeira. O dimensionamento levou em conta a fase de engorda da sardinha verdadeira (de $20 \mathrm{~g}$ até $60 \mathrm{~g}$ ). Foram calculadas as necessidades do sistema para capacidade de $16 \mathrm{~kg} \mathrm{~m}^{-3} \mathrm{e}$ utilizados os seguintes equipamentos: decantador de $0,15 \mathrm{~m}^{3}$, filtro biológico de $1 \mathrm{~m}^{3}$, filtro BEAD pressurizado de $21 \mathrm{~m}^{3} \mathrm{~h}^{-1}$, skimmer com capacidade para $16,95 \mathrm{~m}^{3} \mathrm{~h}^{-1}$ e reatores UV com 40W de potência.
\end{abstract}

Termos para indexação: Aquicultura; Qualidade de água; Tratamento de água.

\section{Sizing of pilot marine aquaculture recirculation system for fish fattening}

\begin{abstract}
The recirculating aquaculture systems (RAS) are alternatives for the growth and maintenance of fish on nets tanks. Researches are being made to develop these systems in commercial scale. The objective of this paper was to design and install a laboratory pilot RAS for Sardinha verdadeira (Sardinella brasiliensis). The sizing of the project focused on the growth phase of the Sardinha verdadeira $(20 \mathrm{~g}$ to $60 \mathrm{~g})$. The needs of the system were calculated for a maximum capacity of $16 \mathrm{~kg} \mathrm{~m}^{-3}$ and used the following equipment: $0,15 \mathrm{~m}^{3}$ decanter, $1 \mathrm{~m}^{3}$ biological filter, pressurized bead filter with capacity of $21 \mathrm{~m}^{3} \mathrm{~h}^{-1}$, a skimmer with capacity of $16,95 \mathrm{~m}^{3} \mathrm{~h}^{-1}$ and UV reactor with $40 \mathrm{~W}$ of potency.
\end{abstract}

Index terms: Aquaculture; Water quality; Water treatment.

\section{Introdução}

A produção global de organismos aquáticos cresceu $25 \%$ entre 2008 e 2017 e foi responsável por 82,1 milhões de toneladas conforme foi divulgado no último relatório The World of World Fisheries and Aquaculture (SOFIA - 2020) (FAO, 2020). Apesar dos desenvolvimentos tecnológicos na aquicultura de peixes marinhos, a aquicultura marinha e costeira produz atualmente muito mais moluscos do que peixes e crustáceos. Segundo Holmer (2010), o cultivo de peixes em tanques-rede, tanto em zonas costeiras como em áreas offshore (mar aberto), traz diversas problemáticas ambientais. Os insumos utilizados podem afetar o ambiente natural, seja pela ração excedente, seja pelo uso de probióticos e antibióticos, bem como pelos resíduos fisiológicos dos animais.
A fuga de animais de cativeiro pode ocasionar a interação com animais selvagens, diminuindo o pool genético da população natural.

Durante os últimos anos tem se observado grande crescimento na indústria aquícola e o desenvolvimento de novas tecnologias de cultivo que trazem maior controle da produção e dos resíduos ambientais, a exemplo dos Sistemas de Recirculação Aquícola - comumente chamados de RAS, sigla em inglês de Recirculating Aquaculture Systems (LEKANG, 2013). Uma das grandes vantagens da produção aquícola em RAS é a redução do uso de água aliada à possibilidade de elaboração de um plano de produção controlada até que o peixe esteja pronto para venda. Isto viabiliza a gestão eficiente da piscicultura e fortalece a competitividade na comercialização. Assim, o objetivo deste trabalho foi de dimensionar e instalar em escala piloto laboratorial um RAS para produção peixes marinhos.

\section{Desenvolvimento}

Os critérios adotados para o dimensionamento deste RAS consistiram em utilizar 4 linhas independentes com $8 \mathrm{~m}^{3}$ cada (Figura 1), montadas no interior de uma estufa agrícola de lona com $210 \mathrm{~m}^{2}$. Cada linha é composta por 4 tanques de fibra de $2 \mathrm{~m}^{3}$ e fundo cônico, com 2 aberturas para drenagem e 1 para abastecimento. A água será bombeada pelas unidades de tratamento: decantador, biofiltro, tanque de equalização, filtro bead e desinfecção UV com vazão de $9,81 \mathrm{~m}^{3} \mathrm{~h}^{-1}$ (Figura 2).

\section{Balanço de massa}

Para dimensionamento utilizouse os índices produtivos da sardinha

Recebido em 19/5/2020. Aceito para publicação em 11/9/2020

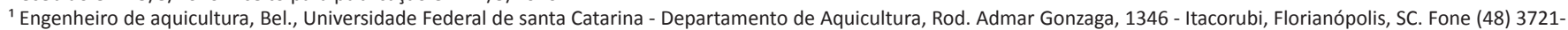
5404, e-mail: guiperuch@hotmail.com.

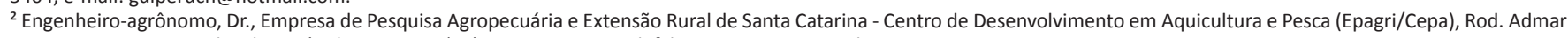
Gonzaga, 1188 - Itacorubi, Florianópolis, SC. Fone (48) 3665-5059, e-mail: fabiano@epagri.sc.gov.br

${ }^{3}$ Engenheira civil, Dr., Universidade Federal de Santa Catarina - Departamento de Aquicultura, Florianópolis, SC. Fone (48) 3721-5404, e-mail: kr.lapa@gmail.com 


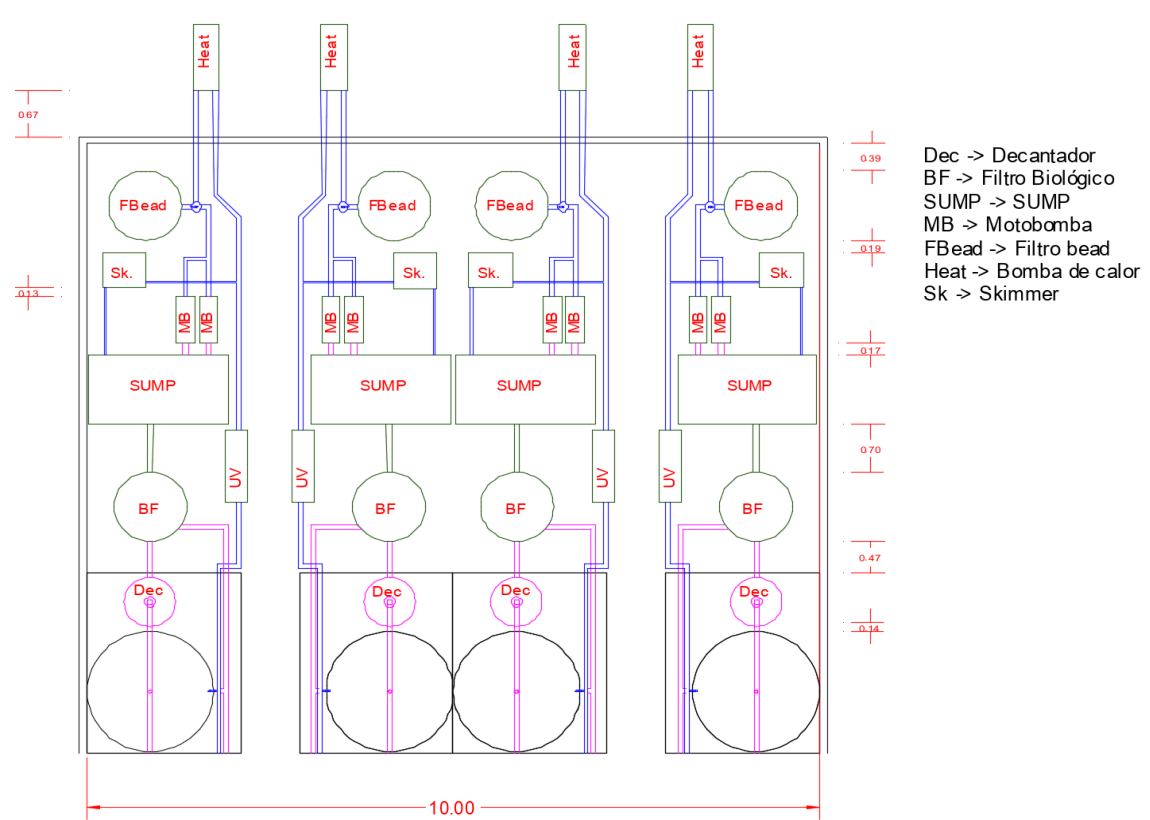

Figura 1. Posição da unidades de tratamento de água. Autor: Luiz G. B. Peruch Figure 1. Water treatment units position. Author: Luiz G. B. Peruch

verdadeira (Sardinella brasiliensis) na fase de engorda, que será o primeiro peixe a ser estudado pela equipe, com a densidade final de $16 \mathrm{~kg} \mathrm{~m}^{-3}(128 \mathrm{~kg}$ por linha), com o índice de alimentação de $4 \%$ da biomassa/dia, prevendo $5,12 \mathrm{~kg}$ de ração (40\% proteína bruta) ao dia, seguindo recomendações de Baloi (2017), entre $2 \%$ e $6 \%$ ao dia. Segundo Timmons \& Ebeling (2010), essa biomassa deve gerar por dia, $1,28 \mathrm{~kg}$ de sólidos totais e $188,42 \mathrm{~g}$ de amônia, além de consumir $0,11 \mathrm{~kg} \mathrm{O}_{2} \mathrm{~h}^{-1}$.

\section{Decantador}

Para o dimensionamento do decantador levou-se em consideração a exis- tência uma incubadora sem uso. Portanto, o decantador foi adaptado a partir de uma incubadora de $0,15 \mathrm{~m}^{3}$. Devido ao seu pequeno volume foi necessário utilizar os limites inferiores indicados na literatura, segundo Timmons \& Ebeling (2010), adotando 15 min como tempo de detenção hidráulica (TDH), o que resultou em $6 \%$ da vazão total do sistema. Sendo abastecido apenas pelo dreno central dos tanques de cultivo, o indicado pelos autores é de $6 \%$ a $15 \%$ de vazão.

\section{Biofiltro}

O biofiltro $\left(1 \mathrm{~m}^{3}\right)$ adotado é do tipo MBBR (Moving Bed Biofilm Reactor),
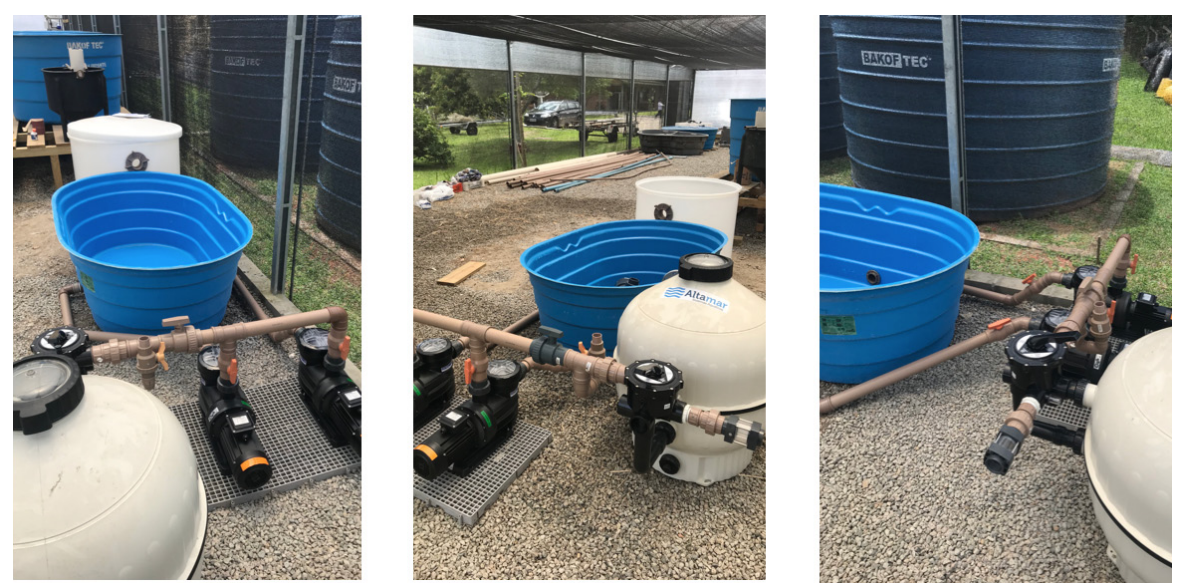

Figura 2. Instalação das unidades de tratamento de água. Foto: Luiz G. B. Peruch

Figure 2. Water treatment units instalation. Photo: Luiz G. B. Peruch

ou seja, de leito móvel com biofilme aderido ao material suporte (mídias de polipropileno - com fração de vazio de $30 \%)$ e receberá $100 \%$ da vazão do sistema vinda do filtro de tambor rotativo. Quando optado pelo decantador, 94\% da vazão será diretamente dos tanques de cultivo (saída lateral) e 6\% será da saída do decantador. Será fornecida aeração constante no fundo através de pratos difusores, com objetivo de manter as mídias flutuantes na coluna de água. O cultivo poderá gerar até $188,42 \mathrm{~g}$ de amônia por dia. Precisará, portanto, de $471 \mathrm{~m}^{2}$ de mídia para fixação da biomassa nitrificante. A mídia utilizada apresenta $680 \mathrm{~m}^{2} \mathrm{~m}^{-3}$, sendo necessário $0,7 \mathrm{~m}^{3}$ de mídia. Todos os cálculos referentes ao biofiltro foram realizados de acordo com as recomendações técnicas encontradas em Timmons \& Ebeling (2010).

\section{Filtro Bead}

Foi incluído também, para realização de testes, um filtro do tipo bead filter pressurizado, com vazão máxima de $21 \mathrm{~m}^{3} \mathrm{~h}^{-1}$, no qual foram utilizadas mídias de polietileno de alta densidade com função principal de filtragem mecânica. É importante ressaltar que o filtro bead pode trabalhar em conjunto com o biofiltro, fazendo função tanto de remoção física como biológica. É necessário saber a especificação da mídia do filtro bead, caso queira usá-lo no tratamento biológico para calcular sua capacidade. Além disso, o tanque de equalização pode servir também para tratamento biológico, sendo necessário existir então aeração no tanque e mídias biológicas (Figura 3).

\section{Aeração}

A aeração deverá ser montada através de uma rede central com divisórias apenas nos pontos finais. Segundo Timmons \& Ebeling (2010) $1 \mathrm{~kg}$ de ração consome $0,5 \mathrm{~kg}$ de $\mathrm{O}_{2}$ por dia. O sistema necessitará de $0,13 \mathrm{~kg}$ de $\mathrm{O}_{2}$ por linha, utilizando uma taxa de folga de $25 \%$. Cada kW do soprador oferece cerca de $0,2 \mathrm{~kg}$ de $\mathrm{O}_{2}$ por dia, necessitando-se $2,67 \mathrm{kWh}$ ou $3,63 \mathrm{cv}$ no total. Portanto, foram utilizados 2 sopradores de $2 \mathrm{cv}$ cada. 

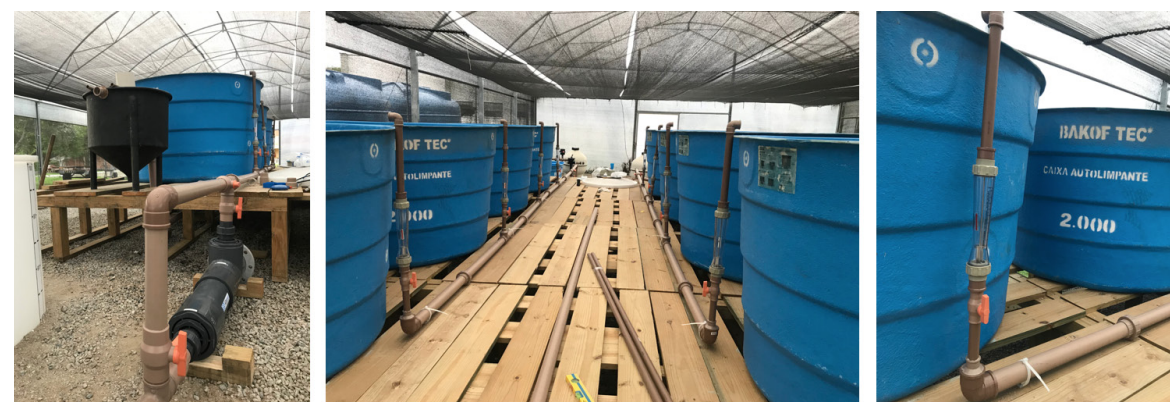

Figura 3. Detalhe do abastecimento de água. Foto: Luiz G. B. Peruch

Figure 3. Water supply detail. Photo: Luiz G. B. Peruch

\section{Skimmer ou fracionador de proteína}

Os Skimmers são equipamentos capazes de remover grande gama de compostos orgânicos da água, como amônia, proteínas surfactantes, entre outros partículas $(>30 \mu \mathrm{m})$. Prevendo experimentos com alta densidade de cultivo, foi dimensionado um skimmer com vazão variando entre 4 a $6 \mathrm{~m}^{3} \mathrm{~h}^{-1}$, dependendo da necessidade de tempo de contato da água com as bolhas, 120 a 90 segundos respectivamente para vazão mínima considerando $5 \%$ do volume total em paralelo (DAVISON, 2018).

\section{Aquecimento}

Para cálculo da potência necessária para os trocadores de calor foi utilizada metodologia proposta por Davidson (2018), chegando à necessidade de 37.225BTUs por linha para manter a água em $26^{\circ} \mathrm{C}$ durante os meses de inverno. Foram então adquiridos trocadores de calor de água salgada com potência de 45.000BTUs.

\section{Desinfecção}

Para desinfecção foi utilizado a dose de $30 \mathrm{mWs} \mathrm{cm}^{-2}$, dose suficiente para matar 99,99\% das bactérias gram-positivas e gram-negativas (KASAl et al., 2002). Utilizando-se a fórmula proposta por Lekang (2013), chegou-se à necessidade de 40W de potência das lâmpadas para atingir a dose estimada.

\section{Consumo de energia}

O consumo de energia foi estimado com as motobombas em $100 \%$ do tempo, trocadores de calor em $50 \%$ do tempo, consumindo $47.073 \mathrm{kWh}$ ao mês, sendo os trocadores de calor responsáveis por $80,6 \%$ do consumo elétrico.

\section{Gerador}

Sistemas de produção dependentes de energia elétrica necessitam obrigatoriamente de um gerador alternativo de energia para momentos de falta de abastecimento. Um curto tempo sem bombeamento e aeração é capaz de matar toda a produção. $O$ cálculo para o dimensionamento do gerador somou todas as cargas dos motores de partida dos equipamentos elétricos que serão utilizados no RAS - Motobombas, compressores de ar, aquecedores, skimmer e reatores UV. Assim, utilizando margem de segurança de $30 \%$, seria necessário um gerador de $130 \mathrm{kVa}$. A utilização de um equipamento eletrônico (soft-starter), capaz de controlar a potência do motor elétrico no instante da partida, reduziria a necessidade para um gerador de $85 \mathrm{kVa}$.

\section{Tubulação e bombeamento}

Para cálculo de tubulação utilizou-se a velocidade máxima do fluido dentro da tubulação de $1,5 \mathrm{~m} / \mathrm{s}$, chegando-se à necessidade de no mínimo $50 \mathrm{~mm}$ de diâmetro nominal. Estas tubulações estão representadas por linhas (cor rosa e azul) no esquema apresentado na Figura 1. Para perda de carga em tubulações foi utilizada a fórmula de Darcy-Weisbach. Chegou-se então à perda de carga de $7,94 \mathrm{~m}$, somada à altura manométrica real do sistema de $1,3 \mathrm{~m}$, totalizando a altura manométrica de $9,24 \mathrm{~m}$. A vazão necessária é de pelo menos $9,81 \mathrm{~m}^{3} \mathrm{~h}^{-1}$. Mas, pensando em cultivos que necessitem vazões maiores para o sistema, foram dimensionadas 2 motobombas de 2 cv por linha, com vazão individual de $23 \mathrm{~m}^{3} \mathrm{~h}^{-1}$, com as curvas do fabricante condizentes com as necessidades. $\mathrm{O}$ sis- tema poderá funcionar com as bombas em regime intermitente ou em conjunto, dependendo da necessidade (ver Figuras 2 e 3 apresentando a montagem das tubulações).

\section{Descarte de efluentes}

Existem quatro pontos de descarte de efluentes: no filtro bead, no skimmer, no decantador ou filtro tambor e diretamente dos tanques através do processo de sifonamento. Esse efluente será direcionado para uma calha de coleta e então devidamente tratado e reaproveitado.

\section{Considerações finais}

Este artigo apresentou a primeira fase de um projeto maior que visa ao estudo em escala piloto de produção de peixes marinhos em Sistema de Recirculação Aquícola. Os próximos passos consistirão em montagem do sistema de abastecimento de água marinha, instalações elétricas e sistema de tratamento de efluentes.

\section{Referências}

BALOI, M.F. Manejo alimentar de juvenis de sardinha-verdadeira Sardinella brasiliensis (Steindachner, 1879). 2017. 111 f. Tese (Doutorado) - Curso de Aquicultura, Universidade Federal de Santa Catarina, Florianópolis, 2017.

DAVISON, A. Recirculating Aquaculture Systems: A guide to farm design and operations. Seattle: Farmfish, 2018. 204p.

FAO. The State of World Fisheries and Aquaculture 2020. In brief. Sustainability in action. Rome. 2020. DOI: https://doi. org/10.4060/ca9231en

HOLMER, M. Environmental issues of fish farming in offshore waters: perspectives, concerns and research needs. Aquaculture Environment Interactions, Odense, p.57-70, 2010.

KASAI, H.; YOSHIMIZU, M.; EZURA, Y. Disinfection of water for aquaculture. Hokkaido: Fisheries Science, 2002. 4p.

LEKANG, O. Aquaculture Engineering. 2. ed. Noruega: Wiley-blackwell, 2013. 433p.

TIMMONS, M.B; EBELING, J.M. Recirculating aquaculture. 2. ed. Ithaca: Cayuga Aqua Ventures, 2010. 489p. 